\title{
A new species of Asphinctopone (Hymenoptera: Formicidae: Ponerinae) from Tanzania
}

\author{
PETER G. HAWKES
}

AfriBugs CC, 379 Bakenkloof Street, Pretoria North, Gauteng Province, 0182, South Africa. Department of Zoology and Entomology, University of Pretoria, Pretoria, Gauteng Province, 0002, South Africa. E-mail: peter.hawkes@afribugs.com

\begin{abstract}
Asphinctopone is a rarely encountered genus of ponerine ants, previously known only from the wet forest zones of West and central Africa. In the most recent revision of the genus Bolton \& Fisher synonymised the three previously described species under $A$. sylvestrii Santschi and described one new but very closely related species, A. differens Bolton \& Fisher. A very distinctive new species, Asphinctopone pilosa $\mathbf{s p .} \mathbf{n}$., is described from Tanzania and represents the first record of this genus from East Africa. Modifications to the diagnosis of the genus and an updated key to the species of Asphinctopone are presented. An analysis of the distribution records of Asphinctopone, using BIOCLIM and DOMAIN as implemented in DIVA-GIS 7.1 to provide preliminary predictions of the potential range of the genus, is also provided.
\end{abstract}

Key words: Africa, Asphinctopone, new species, taxonomy, distribution, Bioclim, Domain

\section{Introduction}

Asphinctopone is a very small genus of rarely encountered Afrotropical ants in the subfamily Ponerinae, tribe Ponerini. The genus was first described by Santschi (1914) with a single species, A. silvestrii. Two more species were subsequently described: A. lucidus (Weber 1949) and A. lamottei (Bernard 1953), the latter originally ascribed by Bernard to a new genus, Lepidopone, but subsequently placed in Asphinctopone by Brown (1953). In a recent revision of the genus, Bolton \& Fisher (2008) synonymised all three previously described species and described one additional species, bringing the total number of described species to two. Of these A. differens (Bolton \& Fisher 2008) is known only from the holotype (collected in the Central African Republic), while A. silvestrii (now including A. lucidus and A. lamottei) has been recorded at least 22 times in West and Central Africa (Bolton \& Fisher 2008, Fisher 2010).

A single Asphinctopone specimen was collected by hand at night during a survey of the Kilindi Forest Reserve (Nguu Mountains, Kilindi District, Tanzania); this was one of 15 sites in Tanzania surveyed during a project investigating ground-dwelling ant and beetle diversity in the Eastern Arc Mountains and Coastal Forests Hotspot of Tanzania and Kenya.

Methods

Sample collection was carried out under COSTECH permit No. 2005-269-NA-2005-57 and the samples exported under the Tanzanian Ministry of Wildlife trophy export certificate No. 48157.

Measurements were taken using a Leica MZ16 stereomicroscope equipped with an ocular micrometer calibrated with a graticule; each measurement was repeated three times and the average is reported. Specimens were photographed using a Leica DFC 420 digital camera connected to the same microscope; montage images were produced using Leica Application Systems (LAS) montage V3.4 and edited with Adobe Photoshop. 
Where available, exact coordinates for previous records of Asphinctopone were obtained from AntWeb (Fisher 2010), otherwise coordinates were estimated based on descriptions of localities in Belshaw \& Bolton (1994), Bernard (1953), Santschi (1914), Watt et al. (2002), Weber (1949) and, where no other source could be found, by estimation of the position of the localities listed in Bolton \& Fisher (2008). 19 unique records for A. silvestrii and one for $A$. differens were identified in this way, in addition to the single record of $A$. pilosa sp. n.

Predicted distributions were generated using BIOCLIM and DOMAIN as implemented in DivaGis 7.1. Nine of the 19 available bioclimatic variables were selected for inclusion on the basis of frequency histograms showing a normal or skewed distribution (see Beaumont et al. 2005). In the latter case the upper or lower tail box was unchecked for BIOCLIM analysis so as to allow this model to include cells with values beyond the observed range in the direction in which the distribution appeared truncated. This was expected at least partly to compensate for the very low number of data points, which was well below the 50-70 considered sufficient by Kadmon et al. (2003) for maximum accuracy of prediction of distributions by BIOCLIM. While possible inaccuracies in estimation of localities might be expected to reduce the reliability of predictions, tests carried out by offsetting all localities $5 \mathrm{~km}$ to the north and east and $5 \mathrm{~km}$ to the south and west showed that, while fine details differed, the overall patterns of the distribution predictions were little affected.

\section{Measurements and indices}

Total Length (TL) Total outstretched length of the ant from mandibular apex to gastral apex; where orientation of specimen does not allow a single measurement to be taken, TL is estimated by summing the lengths of the head (mandibilar apex to cervix), mesosoma (from articulation with cervix to a perpendicular line drawn from the anterior petiolar articulation), petiole length (from anterior to posterior articulations) and gaster (from petiolar articulation to apex)

Head Length (HL) Length of the head measured in full-face view, from the midpoint of the clypeal margin to the midpoint of the occipital margin; where either of these margins is concave the measurement is taken from the midpoint of a line joining the anteriormost portions of the clypeus or the posterior-most portions of the occipital margin

Head Width (HW) Maximum width of the head behind the eyes, measured in full-face view

Cephalic Index (CI) (HW x 100)/HL

Scape Length (SL) Maximum straight-line length of the scape, excluding the basal constriction

Scape Index (SI)

Ocular Diameter (OD)

Ocular Index (OI) (SL x 100)/HW

Maximum diameter of the eye, measured with head in profile view.

Pronotal Width (PW) (OD x 100)/HW

Weber's Length (WL) Diagonal length of the mesosoma in profile, from the junction of the pronotum and the cervical shield, to the posterior basal angle of the metapleuron

Abbreviations of depositories

SAMC Iziko South African Museum, Cape Town, South Africa

\section{Revised key to Asphinctopone species (modified after Bolton \& Fisher 2008)}

1 Metanotal groove only slightly impressed, lateral margins of median clypeal lobe not sharply angulate and not projecting further forward than the median margin. Mandible without a tooth-like process on the inner basal margin. Larger species (HW $0.86 \mathrm{~mm}$ ), entirety of head and mesosoma sculptured and covered with a relatively dense suberect to erect pubescence. (Tanzania) pilosa 
- Metanotal groove deeply impressed, lateral margins of median clypeal lobe sharply angulate and projecting as short denticles slightly anterior to median margin. Mandible with a tooth-like process on the inner basal margin (may be obscured by anterolateral angles of clypeal lobe when mandibles closed). Smaller species (HW 0.62-0.68 mm), head and mesosoma not entirely sculptured nor entirely covered with pubescence; at least the propleura smooth, shining and hairless. (West \& Central Africa) ....

2 With propodeum in profile the dorsum with pubescence extremely scanty, almost absent. Side of propodeum with scattered punctures, the spaces between them usually smooth. (Guinea, Ivory Coast, Ghana, Nigeria, Cameroon, Gabon, Central African Republic) silvestrii

- With propodeum in profile the dorsum with a pelt of erect, weakly curved pubescence. Side of propodeum entirely densely irregularly sculptured. (Central African Republic) ...... differens

\section{Asphinctopone pilosa Hawkes sp. n.}

(Fig. 1, A-E)

Holotype worker. TL 4.48, HL 1.07, HW 0.86, CI 81, SL 0.80, SI 92, OD 0.06, OI 7, PW 0.66, WL 1.38 (all measurements in $\mathrm{mm}$ ).

Medium sized (total length c. $4.5 \mathrm{~mm}$ ) ponerine ant largely conforming to the diagnosis of the genus as presented by Bolton \& Fisher (2008), but with differences in the development of the promesonotal and metanotal grooves, antennal structure and median clypeal lobe as indicated below.

Mandibles smooth and shining, without any sculpture other than coarse scattered hair-pits and with five teeth plus a small denticle between the basal and the second tooth. No tooth-like process on the inner basal margin of the mandible. Eyes very small, comprising a single central ommatidium surrounded by a ring of 6 less distinct ommatidia, positioned distinctly in front of the mid-length of the sides of the head. Ocular diameter less than half the maximum width of the antennal scape. Entire dorsum, sides and ventral surfaces of head with quite coarse punctures (up to $0.03 \mathrm{~mm}$ diameter) with weak irregular sculpture between the punctures on the dorsal and dorso-lateral surfaces. Median portion of the vertex, as well as the sides of the head below the eyes, with larger and more widely spaced punctures. Pubescence on head dense, decumbent and arising both from within the numerous large punctures and from minute hair-pits between these, more sparse on the sides of the head below the eye and on the underside, giving these areas a smoother and more polished appearance. Frontal lobes somewhat more densely and more finely punctulate than remainder of the head; frontal carinae and antennal scrobes absent. In full-face view the sides and the posterior margin of the head very shallowly convex. Antennal scapes when laid back just reaching the occipital margin. Funicular segments $2-10$ broader than long, segments $2-7$ very distinctly so. Funicular segments $8-11$ broader than the preceding 6 , becoming increasingly longer relative to their width and forming an indistinctly 4-segmented club. The terminal funicular segment much longer than broad and distinctly exceeding the combined length of the preceding 5 segments. Clypeus with a projecting median lobe bounded by a rounded obtuse angle on each side, the anterior margin of this lobe flat to very slightly concave laterally and with a broadly rounded median projection. Width of the anterior margin of the projecting clypeal lobe about $0.33 \times \mathrm{HW}$. Several pairs of strong setae and a number of weaker setae present on the clypeus.

Mesosoma with a pattern of sculpturing very similar to that of the head; dorsum and sides of pronotum, mesonotum and propodeum entirely coarsely punctulate and covered with a dense pelt of fine suberect (pronotum and mesonotum) to erect (propodeum) curved pubescence, the katepisternum and the disc of the pronotum with more sparse punctures and pilosity. Ventral surfaces of the mesosoma with dense fine puncturation and reduced pubescence. The punctulate sculpture on the dorsal and lateral mesosoma is overlaid by irregular effaced fine rugulose sculpture everywhere except on the disc of the pronotum and small areas of the lower propleuron and katepisternum. Pronotal humeri broadly rounded; the pronotum with a subglobose appearance in dorsal view. Promesonotal suture moderately impressed and with cross-ribs on the anterior mesonotum, the metanotal groove only slightly impressed and with fewer more widely spaced but much longer cross-ribs. Mesonotum twice as wide as long. Propodeal dorsum narrow, about half the width of the mesonotum and extending horizontally from the level of the metanotal groove to the short upper portion of the declivity, which slopes downward at about $45^{\circ}$ to the junction with the very steep main face of the 
declivity. The propodeal outline thus presents three distinct planes in profile view. Propodeal dorsum about $1.5 \mathrm{x}$ as long as the mesonotum. Declivity of the propodeum the only portion of the dorsal mesosoma lacking sculpture and with greatly reduced pilosity; smooth and weakly shining.
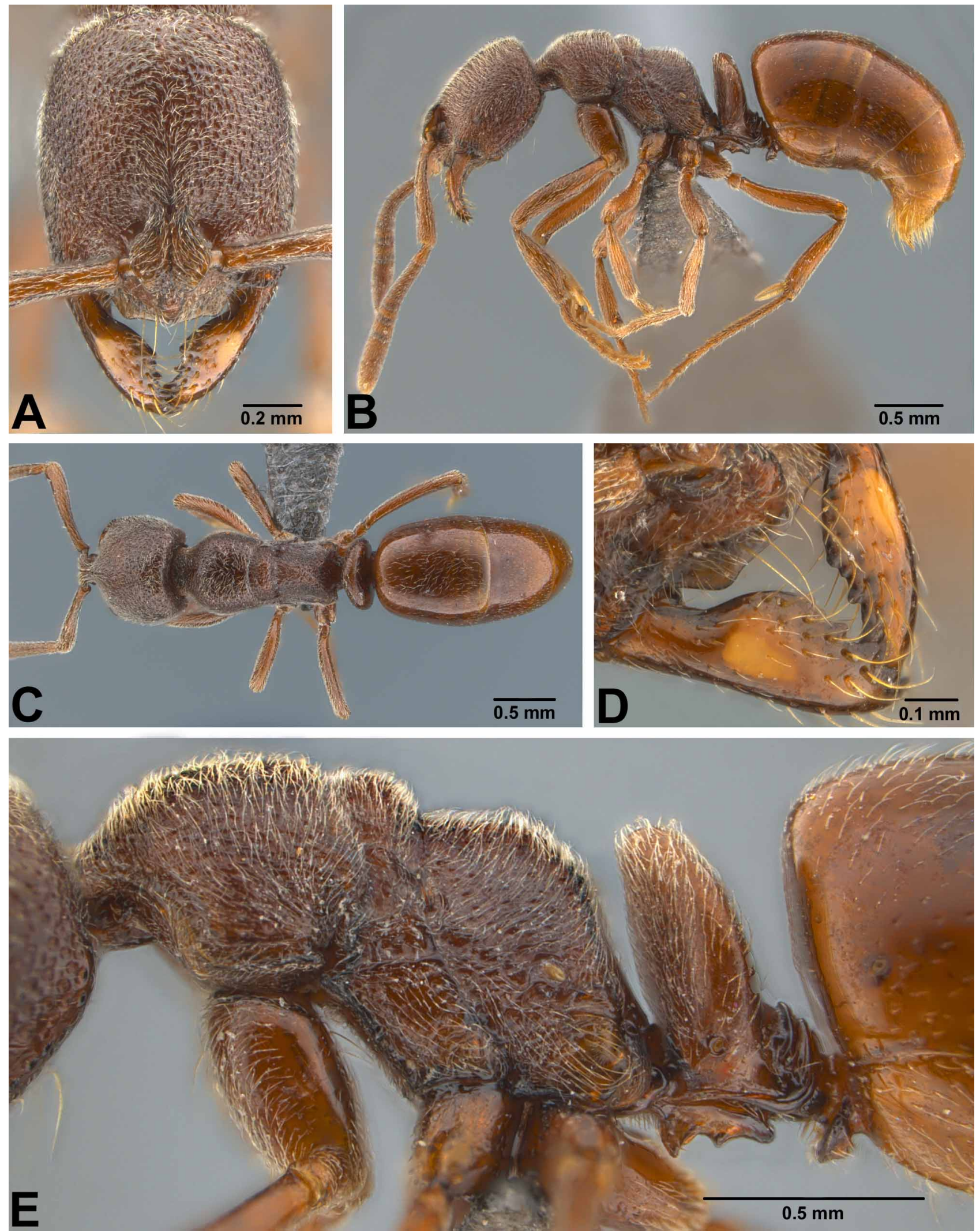

FIGURE 1. Asphinctopone pilosa holotype worker, SAM-HYM-C020683. A, Full face view; B, Lateral view; C, Dorsal view; D, Mandibles, oblique view; E, Mesosoma lateral view. 


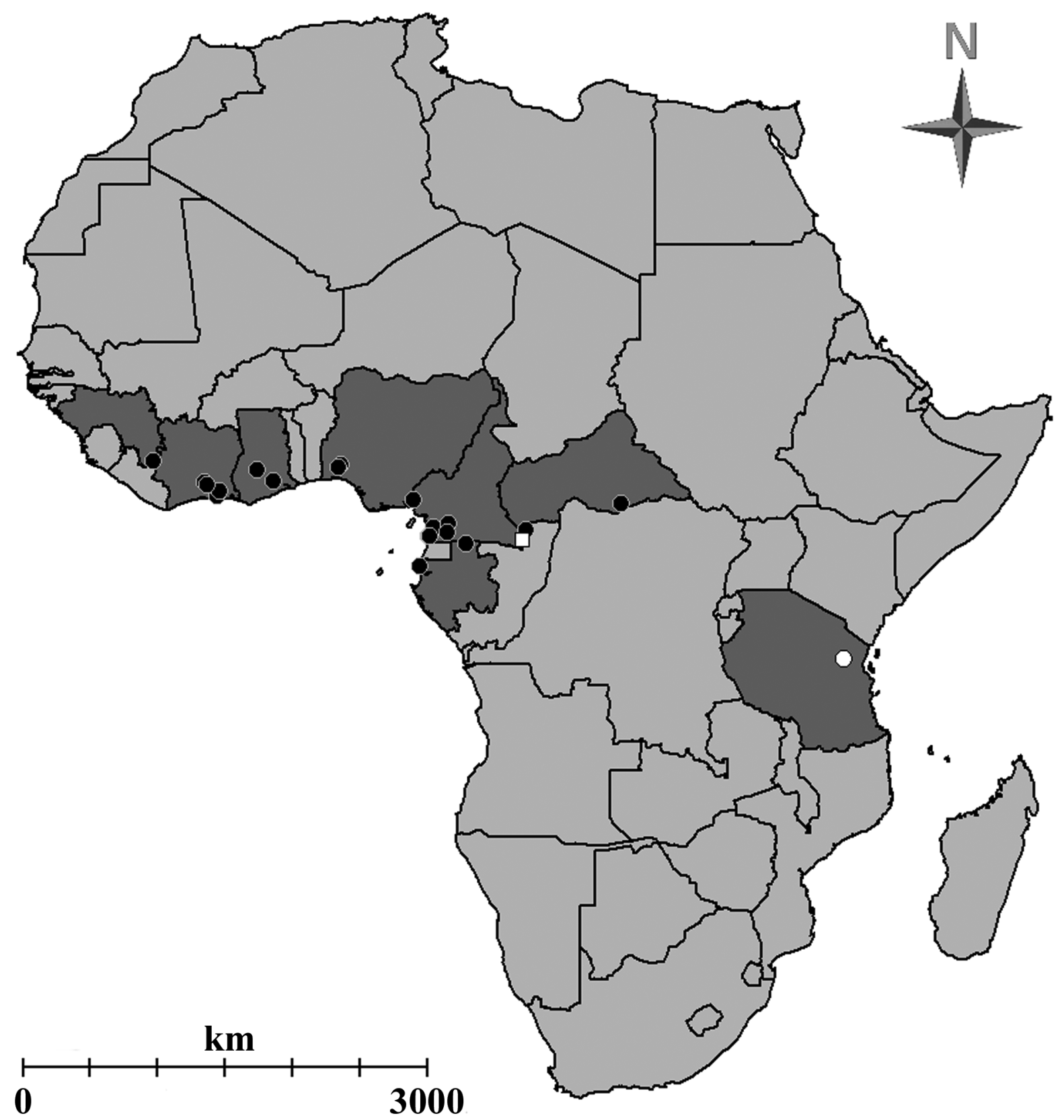

FIGURE 2. Known distribution of Asphinctopone species in the Afrotropical region. Black circles, A. silvestrii; white square, A. differens; white circle, A. pilosa. Dark grey shading indicates countries in which Asphinctopone species have been recorded.

Petiole node high and squamiform, unsculptured apart from minute hair-pits, smooth and weakly shining and with pubescence similar to but less dense than that on the mesosoma. In profile view the anterior and posterior faces of the petiole node almost parallel, only slightly convergent dorsally; in posterior view the dorsal margin forming a bluntly triangular peak. In dorsal view the anterior face shallowly convex, the posterior even more shallowly concave. Subpetiolar process with the complex shape characteristic of the genus.

Gaster unsculptured apart from minute hair-pits (c. $0.005 \mathrm{~mm}$ diameter), pubescence denser but shorter $(0.04 \mathrm{~mm})$ on tergites $2-4$ than on the first gastral tergite $(0.07 \mathrm{~mm})$, much longer and more variable on the sternites. No setae present on gastral tergites 1-4, but a few pairs on each sternite and abundant setae present on the pygidium and hypopygium. Sting stout and slightly upcurved in lateral view, somewhat laterally flattened. 

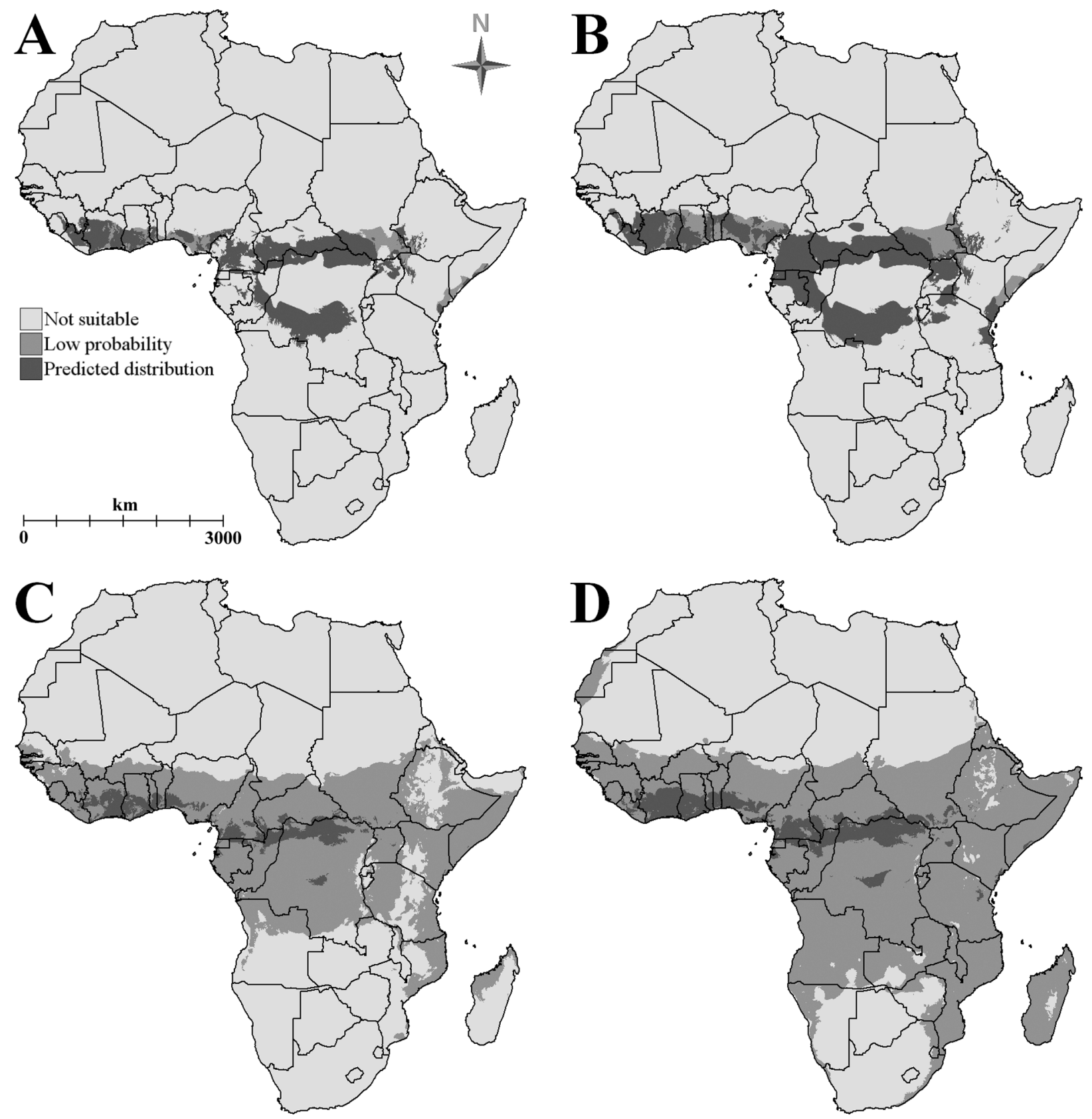

FIGURE 3. Predicted distribution of Asphinctopone from: A, BIOCLIM using A. silvestrii and A. differens localities; B, BIOCLIM using A. silvestrii, A. differens and A. pilosa localities; C, DOMAIN using A. silvestrii and A. differens localities; D, DOMAIN using A. silvestrii, A. differens and A. pilosa localities.

A single (pectinate) spur is present on each tibia, those on the metatibiae much longer $(0.34 \mathrm{~mm})$ than those on the mesotibiae $(0.17 \mathrm{~mm})$, with those on the protibiae intermediate in length $(0.29 \mathrm{~mm})$. All segments of the appendages, including the antennae, with fine appressed pubescence but no standing hairs.

Colour: head and mesosoma dark reddish-brown throughout, the gaster, appendages and mandibles a lighter reddish-brown, with the exception of a small yellowish-brown patch on each mandible.

Holotype worker. Tanzania, Tanga Region, Kilindi Forest Reserve, 1015 m, S 5.57934 E 37.57971, 28.viii.2005, CEPF-TZ-3.4.F34, SAM-HYM-C020683, Primary forest, hand collected (P. Hawkes, J. Makwati, R. Mtana) (SAMC).

Comments. Asphinctopone pilosa is distinctly different from its congeners in many respects; the most obvious differences are 1) its larger size, 2) the stronger and much more extensive sculpturation and denser 
pubescence, 3) the differently shaped mesonotum and propodeum, 4) the far less strongly impressed promesonotal suture and metanotal groove, 5) the terminal four, rather than three, antennal segments forming a weak club, 6) the more strongly squamiform petiole, which is also more arcuate in dorsal view, 7) the lessdeveloped clypeal structure (in sylvestrii and differens the median clypeal lobe is bounded by distinct sharp angles, is distinctly though shallowly concave on either side of the more acutely rounded median projection and is relatively broader at about $0.40 \mathrm{x} \mathrm{HW}), 8$ ) the lack of a tooth-like process on the inner basal margin of the mandible and 9) its darker colour.

Etymology: The specific epithet pilosa refers to the pubescence which is much denser on the head and mesosoma than in all previously described species of the genus.

Habitat. The Kilindi Forest Reserve (5395 ha) lies about $140 \mathrm{~km}$ inland and comprises a small area of forest on an isolated double-peaked mountain surrounded by miombo woodland; the survey site was located at about $1000 \mathrm{~m}$ altitude on a mainly flat area on the saddle between the two peaks. The forest canopy here was estimated to average about $25 \mathrm{~m}$ high, with some emergent trees of 35-40 m; closure of the canopy was estimated at approximately $70-80 \%$. Previous disturbance and tree felling by informal gold miners was evident and numerous young trees and climbers were observed in partially cleared areas. Soils along the survey transect were mainly of sandy loam texture (with some patches of sandy clay loam), a $\mathrm{pH}$ of 8.0-8.5 and with a thin (1-2 cm depth) leaf litter layer ranging from $25 \%$ to $100 \%$ cover. The survey was carried out toward the end of the cool dry season in late August 2005; soil temperatures averaged $16^{\circ} \mathrm{C}$ at a depth of 10 $15 \mathrm{~cm}$, with soil moisture at the same depth averaging 11.9\%. The Asphinctopone pilosa specimen was, however, collected in a microhabitat with higher moisture levels and a much thicker litter layer than average for the site. Humidity during the three-day site visit ranged from $63.4 \%$ to $99.3 \%$ (the latter during an unseasonal rainstorm) and averaged $81.4 \%$, while the temperature varied from $12.0-22.5^{\circ} \mathrm{C}$.

Climate. Detailed climatic data are not readily available for all of the localities from which other Asphinctopone specimens have been collected, but inspection of Worldclim (version 1.3) 2.5 arc-minute extrapolated climate data (Hijmans et al. 2005) downloaded via http://www.diva-gis.org/climate provides some indication of conditions within the known distribution (see Figure 2) of the genus. Most of the localities exhibit low seasonal variation in temperature and precipitation. The A. pilosa locality was an outlier in respect of some of the bioclimatic variables (it has the highest temperature seasonality, lowest mean annual temperature and lowest mean temperature of the driest quarter), but in most variables was similar to previously recorded Asphinctopone localities.

Modelling of habitat suitability and predicted distribution. Of the nine bioclimatic variables selected for inclusion in the model, two showed a close to normal and one a truncated normal distribution, while six were strongly skewed. For three of the variables with skewed distributions (min. temperature of coldest month, mean temperature of coldest quarter and mean temperature of driest quarter) estimates from visual inspection indicated that less than 5\% of the entire African continent has values higher than the modal value of the Asphinctopone localities. This suggests that intolerance to low temperatures may be a significant factor influencing distribution. Test predictions in BIOCLIM using all of the 19 bioclimatic variables or the selected subset of nine without the tail box adjustment gave implausible results with most (14-16 of 19) recorded $A$. silvestrii localities placed at or near $(<10 \mathrm{~km})$ an edge of the predicted distribution; using the adjustment and the selected variable subset gave far more probable results, with the majority (13) of these records falling well within the predicted distribution.

In addition to the seven countries (Cameroon, Central African Republic, Gabon, Ghana, Guinea, Ivory Coast and Nigeria) from which A. silvestrii and A. differens have been recorded to date, BIOCLIM modelling predicted a reasonable probability (see Figure 3A) that one or both of these species could occur in a further 12 African countries (Angola, Benin, Democratic Republic of the Congo, Ethiopia, Equatorial Guinea, Kenya, Liberia, Republic of the Congo, Sierra Leone, Sudan, Toga and Uganda). However, in some cases the distribution within these countries is expected to be very limited (e.g. to the far north-east in Angola, the southern Sudan and western Ethiopia). Inclusion of the A. pilosa locality in the model resulted in the predicted suitable area in many of these countries increasing substantially (see Figure 3B) and also in the inclusion of parts of north-western Tanzania. While this may not be a realistic expansion of the predicted 
ranges of A. silvestrii and A. differens, it could indicate the possibility of additional Asphinctopone species in areas beyond the ranges of the known species.

It is recognised that BIOCLIM often under-predicts species distributions (Ward 2007), but the use here of a selected subset of bioclimatic variables and adjusting tail checkboxes resulted in a predicted distribution greater than the core area predicted by DOMAIN (Figure 3C and 3D). The large non-core area mapped by the latter model illustrates a low predicted probability of occurrence, so the apparently very large predicted distributions in Figure 3C and 3D should perhaps be viewed with some scepticism, especially as the latter includes some very improbable areas such as a large portion of the Namib desert.

\section{Discussion}

Taxonomy. While Asphinctopone pilosa displays certain characters that do not fit the diagnosis of the genus as presented by Bolton \& Fisher (2008), there can be no doubt that it belongs in this genus; several sections of their diagnosis should therefore be modified slightly to read as follows (characters in italics were considered autapomorphic by Bolton \& Fisher, 2008; the numbering of characters by these authors has been retained for ease of reference):

4 Basalmost tooth is at the rounded basal angle; basal margin shallowly convex.

6 Clypeus complex: in full-face view the median portion projects anteriorly as a broad lobe which terminates in a rounded to distinct angle on each side; these angles overlap the basal margins of the mandibles; the anterior clypeal margin has a median rounded projection, on each side of which the anterior margin is flat to shallowly concave; above the median projection the central portion of the clypeus forms a narrow ridge to the frontal lobes.

8 Antenna with 12 segments, gradually incrassate apically, with the terminal three or four segments forming a weak club, apical antennomere hypertrophied, longer than the five preceding segments together.

9 Promesonotal suture moderately to deeply impressed, cross-ribbed on extreme anterior mesonotum; metanotal groove slightly to deeply impressed and cross-ribbed; mesonotum clearly to conspicuously isolated by these impressions (worker only).

The lack of a) a deeply impressed promesonotal suture and b) a tooth-like process on the basal mandibular margin in Asphinctopone pilosa reduces the degree to which Asphinctopone is distinguished from the melanaria-group species of Pachycondyla (see Bolton \& Fisher 2008). However, numerous distinguishing characters as described by Bolton \& Fisher (2008) remain to clearly separate these taxa: in the melanariagroup species of Pachycondyla the mandible is triangular to elongate-triangular rather than oblique, there are more than 6 teeth on the apical mandibular margin, the apical antennomere is not hypertrophied, the anterior clypeal margin is simple, the promesonotal suture is not cross-ribbed, the mesotibiae and metatibiae each have two spurs, the petiole sternite has a simple posterior structure, the helcium and prora are not modified as in Asphinctopone and presclerites are present on the second gastral segment (the apparently derived helcium structure has not yet been confirmed in A. differens or A. pilosa as this would require dissection of their respective unique holotypes).

Habitat and distribution. Asphinctopone are very rarely encountered; whether this is due to actual rarity of members of the genus, or to a secretive and perhaps deeply subterranean lifestyle, is unknown. The significance of the A. pilosa specimen was not recognised at the time of collection, but the location (an accumulation of moist leaf litter at the base of a large sloping rock outcrop) was revisited the following day as it was observed to be moister and more productive in terms of ponerine ant species than its surroundings. However no further specimens of Asphinctopone were found in the additional samples collected here, or in any of the other standardized pitfall, leaf litter or hand-collected samples. No other Asphinctopone specimens were found amongst the 67208 ants collected and processed during the course of the project. This confirms the rarity of encounter of members of the genus as discussed by Bolton \& Fisher (2008), who noted that 
Belshaw \& Bolton (1994) reported finding only five Asphinctopone specimens in a total of 43824 ants collected in a survey of leaf litter in Ghana.

That the only known specimen of A. pilosa was collected during the evening (between 19:30 and 20:00), while no further specimens were found in the same area during daytime, suggests the possibility that they are nocturnal, but this is clearly pure conjecture at this stage given the limited information available.

The distribution of Asphinctopone previously appeared to be limited to the moist forest zones of West and Central Africa; its discovery in the Kilindi Forest Reserve represents a more than $1600 \mathrm{~km}$ eastward extension of the known range of the genus, and the site is approximately $2000 \mathrm{~km}$ from the closest previously recorded Asphinctopone locality. While subjective predictions (Guénard et al. 2010) suggest that Asphinctopone should occur in some additional counties (Benin, Equatorial Guinea, Liberia, Sierra Leone and Toga), the BIOCLIM modelling presented here indicates that a much larger range is likely, with presence expected in at least a further seven countries (Angola, Democratic Republic of the Congo, Ethiopia, Kenya, Republic of the Congo, Sudan and Uganda) in addition to the confirmed presence in Tanzania.

Although some habitat predicted to be suitable for A. silvestrii and A. differens is shown in the coastal regions of Kenya, northern Mozambique and Somalia (Figure 3), the broad band of unsuitable habitat isolating these areas from the remainder of the predicted distribution of these species suggests that they are unlikely to occur here. It is far more likely that A. pilosa will be found in these areas. Other areas which appear bioclimatically suitable but geographically improbable for Asphinctopone are the Comoros Islands and parts of Madagascar.

Exclusion of the bulk of the central Congo Basin from the BIOCLIM predictions appears to be due largely to the very low precipitation seasonality and (obviously correlated) high precipitation of the driest quarter, as well as the very low temperature seasonality of this region. A trial run showed that inclusion of a single positive point within the central Congo basin would result in BIOCLIM including the entire basin in the predicted distribution. This part of the map could therefore change significantly with very little additional data. It is also important to note that I have here attempted to model the potential distribution of a genus using data almost entirely (90\%) on only one of three Asphinctopone species; there is thus little scope for influence of the preferences of the other two species, nor any other as yet undiscovered species, on the extent of the predicted distribution. It is therefore possible that the distribution of Asphinctopone will eventually be found to be much greater than suggested here by BIOCLIM, and maybe even to cover a substantial portion of the low probability area predicted by DOMAIN, but it is hoped that this exercise will help to focus efforts on finding additional material in the most likely areas.

It should be kept in mind that while occurrences of specimen records in areas with particular bioclimatic conditions may appear to indicate a preference for such conditions, it is also possible (especially in the case of cryptic taxa such as Asphinctopone) that this is an artefact of the conditions providing greater probability of discovery. For example, in areas with low precipitation seasonality and/or higher minimum temperatures, subterranean species may spend more time closer to the soil surface and therefore be more likely to be collected. A much broader Asphinctopone distribution than suggested by BIOCLIM is thus possible, and could be revealed by more intensive searches using deeper excavation than currently employed in most ant surveys.

\section{Acknowledgements}

The survey during which the Asphinctopone specimen was collected formed part of a project investigating ground-living ant and beetle diversity in Tanzania, funded by the Critical Ecosystem Partnership Fund (CEPF), which is a joint initiative of Conservation International, the Global Environment Facility, the Government of Japan, the MacArthur Foundation and the World Bank. A fundamental goal of CEPF is to ensure civil society is engaged in biodiversity conservation. Thanks are due to Joas Makwati and Renald Mtana whose assistance with the fieldwork was invaluable, as well as Dr Bruno Nyundo of the University of Dar es Salaam for logistical assistance and Barry Bolton for his advice and encouragement and for providing valuable comments on a draft of this manuscript. 


\section{References}

Beaumont, L.J., Hughes, L. \& Poulsen, M. (2005) Predicting species distributions: use of climatic parameters in BIOCLIM and its impact on predictions of species' current and future distributions. Ecological Modelling, 186, 250-269.

Bolton, B. \& Fisher, B.L. (2008) The Afrotropical ponerine ant genus Asphinctopone Santschi (Hymenoptera: Formicidae). Zootaxa, 1827, 53-61.

Brown, W.L.,Jr. (1953) Characters and synonymies among the genera of ants. Part II. Breviora, 18, 1-8.

Belshaw, R. \& Bolton, B. (1994) A survey of the leaf litter ant fauna in Ghana, West Africa (Hymenoptera: Formicidae). Journal of Hymenoptera Research, 3, 5-16.

Bernard, F. (1953) La reserve naturelle integrale du Mt Nimba. XI. Hyménoptères Formicidae. Mémoires de l'Institut Français d'Afrique Noire, 19, 165-270.

Fisher, B.L. (2010) Antweb. Available from http://www.antweb.org (accessed 12 February 2010)

Hijmans, R.J., Cameron, S.E., Parra, J.L., Jones P.G. \& Jarvis, A. (2005) Very high resolution interpolated climate surfaces for global land areas. International Journal of Climatology, 25, 1965-1978.

Kadmon, R., Farber, O. \& Danin, A. (2003) A systematic analysis of factors affecting the performance of climatic envelope models. Ecological Applications, 13(3), 853-867.

Santschi, F. (1914) Formicides de l'Afrique occidentale et australe du voyage de Mr. le Professeur F. Silvestri. Bollettino del Laboratorio di Zoologia Generale e Agraria della R. Scuola Superiore d'Agricultura, 8, 309-385.

Ward, D.F. (2007) Modelling the potential geographic distribution if invasive ant species in New Zealand. Biological Invasions, 9, 723-735.

Watt, A.D., Stork, N.E. \& Bolton, B. (2002) The diversity and abundance of ants in relation to forest disturbance and plantation establishment in southern Cameroon. Journal of Applied Ecology, 39, 18-30.

Weber, N.A. (1949) New Ponerine Ants from Equatorial Africa. American Museum Novitates, 1398, 1-9.

Guénard, B., Weiser, M.D. \& Dunn, R.R. (2010) Ant Genera of the World. Available from http://www.antmacroecology. org/ant_genera/index.html (accessed 12 February 2010). 